\title{
VISUAL PERCEPTION AND NATURAL ILLUMINATION
}

\author{
Richard F. Murray ${ }^{1}$ and Wendy J. Adams ${ }^{2}$ \\ ${ }^{1}$ Department of Psychology and Centre for Vision Research, York University \\ ${ }^{2}$ School of Psychology, University of Southampton
}

Corresponding author:

Richard F. Murray

Centre for Vision Research

York University

4700 Keele Street, LAS 0009

Toronto, Ontario

Canada M3J 1P3

tel (416) $736-2100 \times 23025$

fax (416) 736-5857

rfm@yorku.ca 


\begin{abstract}
We review recent work on the role of natural illumination in human vision. We discuss research showing that visual perception depends on stable statistical properties of natural light in order to solve the under-constrained problem of estimating the shape, colour, and material properties of surfaces and objects. We focus on assumptions about the distribution of luminous flux over 3D directions and spatial locations. We also review work showing that implicit assumptions about lighting colour may explain the remarkable individual differences in percepts of \#thedress. We conclude by discussing the unsolved problem of how the human visual system represents lighting, and outlining promising directions for future work that have been made possible by recent advances in physically based rendering and light measurement.
\end{abstract}

\title{
HIGHLIGHTS
}

- Human vision relies on statistical regularities in natural lighting

- We use assumptions about lighting direction, diffuseness, and more complex properties

- Individual differences in \#thedress reflect assumptions about lighting colour

- Progress in this area is supported by advances in rendering and light measurement 


\section{VISUAL PERCEPTION AND NATURAL ILLUMINATION}

\section{Introduction}

Illumination in the natural environment has shaped the evolution of the human visual system (Cronin, Johnsen, Marshall, \& Warrant, 2014), and it plays a key role in circadian rhythms (Duffy \& Czeisler, 2009), affect and mental health (Magnusson \& Boivin, 2003), social organization (Brox, 2011), and aesthetics (Cuttle, 2015). Less obvious but equally important is how human vision relies on stable properties of lighting in order to perceive fundamental object properties such as shape and colour. Visual perception is a highly under-constrained problem, in that a typical retinal image can be produced by many different combinations of lighting, shape, and materials (e.g., Belhumeur, Kriegman, \& Yuille, 1999). As a result, the visual system must use statistical knowledge (usually implicit) about typical scene properties to infer the external world that a retinal image is most likely to be depicting. It has long been understood that ecological statistics play a central role in human vision (e.g., Brunswik \& Kamiya, 1953; Gibson, 1979), and recent advances in measuring devices and computational modelling have made it possible to investigate this role quantitatively (Geisler, 2008).

Discovering how vision relies on illumination statistics has been an important part of this story.

Here we review recent work on key properties of illumination, mostly focusing on the distribution of light over directions and spatial locations, as well as illumination colour. We examine how human vision exploits these regularities to estimate the shape and material composition of objects. We conclude by noting some unsolved problems and outlining promising directions for future work. 


\section{Perceptually important properties of illumination}

\section{Illumination direction}

Objects are illuminated by both primary light sources and light reflected from nearby surfaces, so the complete lighting conditions at a point in space are usually complex. We can describe the lighting at a given point with an illumination map, a real-valued function of spherical coordinates $f(\theta, \phi)$ that reports the luminance in each direction $(\theta, \phi)$ at that point (Gershun, 1939; Debevec, 1998). Just as a 2D image can represented as a sum of sinusoids via Fourier analysis, an illumination map can be represented as a sum of spherical harmonics (Figure 1), and this representation is useful for quantifying several basic properties of lighting.

One of the simplest and most important properties of lighting is its direction, the 3D orientation at which a $2 \mathrm{D}$ surface patch receives the greatest luminous flux. This is also the $3 \mathrm{D}$ orientation of the first-order spherical harmonic representation (Figure 1). In natural scenes there is a strong tendency for light to come from above, and classic ambiguous figures show that people tend to see shaded stimuli as if they are illuminated from overhead (Metzger, 1936/2006; Ramachandran, 1988a; see Figure 2). In Bayesian terms, people have a "lightfrom-above prior" (Adams, Graf, \& Ernst, 2004) that modulates the perception of shape, reflectance, and visual search for shaded targets (Adams, 2007).

The light-from-above prior appears to be at least partially learned, with a surprisingly long time course. It emerges by seven months (Granrud, Yonas, \& Opland, 1985) and continues to develop at least until ten years of age (Thomas, Nardini, \& Mareschal, 2010). Even for adults, robust lighting direction cues from shading and shadows can have a stronger influence on shape-from-shading judgements than the light-from-above prior, reflecting how Bayesian priors are combined with information from sensory cues (Morgenstern, Murray, and Harris, 2011; see Figure 2). Furthermore, the light-from-above prior reflects recent experience with the environment: when an observer is immersed in a visual-haptic environment where the illumination direction differs from their prior, they update their prior accordingly (Adams et al., 
2004). Such learning may be context-specific, as people can learn at least two distinct lighting direction priors for use in different visual environments (Kerrigan \& Adams, 2013).

\section{Illumination diffuseness}

Another important property of lighting is diffuseness, the degree to which luminous flux is distributed across the full range of 3D directions, e.g., diffuse light on a cloudy day vs. more directed light on a sunny day. In a spherical harmonic expansion (Figure 1), diffuseness is shown by the magnitude of the zero-order component -- which is constant in every direction -relative to the higher-order components. There are several ways of making this definition more precise, and measures of diffuseness are a topic of ongoing research (Xia, Pont, \& Heynderickx, 2017a, 2017b).

Lighting diffuseness plays a key role in lightness constancy, the ability to perceive greyscale surface reflectance correctly under a wide range of lighting conditions. Diffuseness affects the relationship between surface reflectance, surface orientation, and image luminance: under directional light, the luminance of a surface depends on its orientation relative to the dominant light source, whereas under highly diffuse light, luminance is approximately the same at all orientations. Thus mis-estimating diffuseness should result in errors in perceived reflectance. In fact, human observers do show deviations from lightness constancy that are consistent with an overestimation of diffuseness (Boyaci, Maloney, \& Hersh, 2003; Bloj et al., 2004). Furthermore, illumination in natural environments is typically more diffuse than in the experiments where these partial failures of lightness constancy were observed (Morgenstern, Geisler, \& Murray, 2014). This suggests that people may learn a prior for high diffuseness in natural environments, and that this prior influences lightness judgements in artificial environments even when diffuseness cues such as shadow penumbra provide information about the atypical lighting conditions. 
In addition to making the probably implicit judgements of diffuseness that guide lightness perception, people can, to some extent, make explicit judgements of diffuseness. These judgements are often inaccurate, but they are monotonically related to the true diffuseness level in the scene (Pont \& Koenderink, 2007; Xia, Pont, \& Heynderickx, 2017c). People tend to overestimate diffuseness, especially in scenes with relatively directional light, which is consistent with the evidence cited above that people have a prior for diffuse light (Koenderink et al., 2007; Xia et al., 2017c). People also tend to overestimate diffuseness when light comes from the viewer's direction (the "diffuseness-direction ambiguity"; Pont \& Koenderink, 2007; Xia et al., 2017c) but this bias seems to have a different origin: in images that are illuminated either diffusely or from the direction of the viewer, light is distributed more evenly over a wide range of surface orientations, and so these two lighting conditions produce images that are physically quite similar, and hence confusable.

\section{Higher order features of illumination}

Natural illumination tends to be complex, but it follows certain regularities. For example, in addition to luminance increasing with elevation (i.e., light from above), the luminance distribution tends to be highly skewed (with a few very bright small regions) and follows a $1 / f^{2}$ power distribution (Dror, Willsky \& Adelson, 2004; Adams, Kucukoglu, Landy \& Mantiuk, 2018).

Figure 1 shows matte and glossy objects rendered in an illumination environment that has been approximated with various orders of spherical harmonics. High frequency variations in illumination (beyond 2nd order) have little effect on the image of a matte, convex object (Basri \& Jacobs 2001; Ramamoorthi \& Hanrahan, 2001). However, cast shadows become sharper when high frequencies are included, and thus even in a Lambertian world, higher-order illumination structure has implications for image segmentation: cast shadow boundaries must be distinguished from other types of image edges, such as object boundaries (Khan, Bennamoun, Sohel \& Togneri, 2016; Ehinger, Adams, Graf, \& Elder, 2017). Glossy objects change 
dramatically in appearance as higher-order components are introduced, which modify the pattern of specular highlights.

The influence of illumination on gloss perception is illustrated by failures of gloss constancy when the illumination changes (Olkkonen \& Brainard, 2010; Pont \& te Pas, 2006; Motoyoshi \& Matoba, 2012). To understand which features of illumination are important for gloss perception, we can probe the perceptual effects of different illumination manipulations. For example, changing the luminance skew, or luminance contrast of the illumination environment modulates the apparent gloss of the illuminated object (Adams et al., 2018). A glossy sphere rendered under phase scrambled illumination (or $1 / \mathrm{f}$ noise) appears to be a 2D matte object, highlighting the importance of illumination structure (Fleming, Dror, \& Adelson, 2003).

When the illumination is modified, the effects on gloss perception can be understood in terms of resultant changes to specular highlights: perceived gloss increases with the brightness, sharpness, and coverage of highlights (Hunter, 1937; Marlow \& Anderson, 2013; Marlow, Kim, \& Anderson, 2012). However, Bayesian theories of perception suggest that observers should infer an object's gloss from its specular highlights (in line with how these are generated under natural illumination), but also take account of sensory evidence about the current illumination. When provided with contextual information about the illumination conditions, observers do not become not fully gloss constant, but their gloss judgements are modulated by some statistics of the visible illumination environment (Adams et al., 2018).

\section{Illumination distribution over space}

Mury, Pont, and Koenderink (2007, 2009a, 2009b) measured spatial variations in illumination within natural scenes, and found that lower order illumination components (which typically encompass more energy) are relatively stable across spatial locations, and reflect coarse scene geometry. Scene geometry causes greater spatial variation in illumination under 
sunny conditions, via cast shadows and inter-reflections. Low-order illumination structure can change abruptly, however, such as in a forest on a sunny day, when spatially intermittent overhead foliage modulates both low and high order components.

To probe our ability to estimate spatially varying illumination, observers have been asked to directly adjust the illumination of a planar patch or spherical 'probe', or to estimate the reflectance or shape of objects. Observers can adjust the intensity, direction and diffuseness of illumination on spherical probes at different locations with reasonable accuracy within a simple photographed scene (Koenderink et al., 2007) or in real scenes (Xia, Pont, \& Heynderickx, 2014, 2017c). Light field estimates are somewhat simplified compared to ground truth structure, and biased toward diverging light fields (i.e., light radiating from a source; Kartashova, Sekulovski, de Ridder, te Pas, \& Pont, 2016) but do represent different 'light zones' (Kartashova, de Ridder, te Pas, \& Pont, 2018).

Gilchrist (1977) demonstrated that observers' reflectance judgements can change dramatically depending on the perceived location, and therefore the perceived local illumination of a surface patch. Although he emphasised discrete zones, or 'frameworks' for reflectance estimation (Gilchrist et al., 1999), subsequent studies have shown that observers can compensate for smooth changes in illumination (e.g., Ikeda et al., 1998; Snyder et al., 2005), using cast shadows, shading and specular highlights to infer and interpolate illumination conditions (Boyaci, Doerschner, \& Maloney, 2006).

When perceived illumination is inferred from the perceived shape of simple shaded disks (similar to Figure 2), a prior for spatially invariant illumination is apparent: although the perceived shape (convex vs. concave) of an array of such disks can be perceptually bistable, all are perceived in accordance with the same illumination direction at any one time (Ramachandran, 1988b). This prior can be overruled by additional information (e.g., shape information from stereopsis or touch; Adams, Kerrigan, \& Graf, 2010), or even the presence of specular highlights, which promote a convex interpretation (Adams \& Elder, 2014). 
van Doorn and colleagues (van Doorn, Koenderink, \& Wagemans, 2011; van Doorn, Koenderink, Todd, \& Wagemans, 2012) presented annuli of shaded disks, arranged to approximate complex light fields, including diverging (from an invisible central illuminant), converging (as though objects are surrounded by a ring of illumination) or rotational (simulated illumination follows a circular path). Observers' shape perception did not follow the simulated converging or rotational illumination patterns, but the use of simple disks with linear luminance gradients (rather than more realistic rendering) may have affected observers' ability to perceive these more unusual illumination configurations.

Wilder, Adams, and Murray (2019) demonstrated that observers can estimate shape from shading for an undulating surface when illumination direction varies rapidly from place to place. This work highlights a striking disconnect between implicit and explicit representations of illumination: observers were able to accommodate large changes in illumination when judging shape, but they were unable to explicitly detect these changes. Similarly, Ostrovsky, Cavanagh, and Sinha (2005) showed that observers are surprisingly insensitive to large inconsistencies in illumination across different objects in a scene. In addition, observers' explicit estimates of illumination can differ substantially from the implicit illumination estimates that would account for their reflectance judgements (Rutherford \& Brainard, 2002). Although accounting for illumination is critical for accurate perception of shape and reflectance, we rarely need to explicitly estimate or report it.

\section{Illumination colour: \#thedress}

The image known as \#thedress gives a striking illustration of the fact that visual perception is a subjective inference based on deeply ambiguous stimuli. People disagree strongly about the colour of the depicted dress: some see a white dress with gold trim, while others see a deep blue dress with black trim. Low-level factors may contribute to these large individual differences, including differences in pre-retinal filtering and spectral sensitivities 
(Brainard \& Hurlbert, 2015; Rabin, Houser, Talbert, \& Patel, 2016; Vemuri, Srivastava, Agrawal, \& Anand, 2018), although a twin study indicates individual differences in perceived dress colour are mostly due to environmental rather than genetic factors (Mahroo et al., 2017). The explanation supported by most research is that people make different estimates of the lighting conditions in this picture, and so when colour constancy mechanisms discount the lighting, very different estimates are produced of the material colour of the dress (Brainard \& Hurlbert, 2015). (We have not reproduced the dress image here due to copyright restrictions, but for the original image and the chromatic variants of it discussed below, see Gegenfurtner, Bloj, and Toscani (2015).)

A key property of the dress image seems to be that its chromaticities fall on the same axis in colour space as natural daylight (Figure 3). If the image is rotated in colour space, it is much less ambiguous (Gegenfurtner et al., 2015). Even if inverted in colour space, it is less ambiguous: this transformation preserves chromaticities, but changes the correlation between chromaticity and luminance so that it no longer matches that of natural daylight, where deeper blues tend to be darker (Gegenfurtner et al., 2015; Winkler, Spillmann, Werner, \& Webster, 2015). This is intriguing, but a complete understanding of how the daylight axis contributes to individual differences in the illusion will require a more thorough computational analysis (Brainard \& Hurlbert, 2015).

The most direct evidence that lighting estimates play an important role in the illusion is that the dress image is much less ambiguous when shown with strong lighting cues (LaferSousa, Hermann, \& Conway, 2015; Witzel, Racey, \& O’Regan, 2017; but see Dixon \& Shapiro, 2017). Furthermore, people who perceive the dress colour differently also perceive different lighting chromaticities in the image (Toscani, Gegenfurtner, \& Doerschner, 2017; Uchikawa, Morimoto, \& Tomohisa, 2017). Interestingly, after naive observers see the dress image in a context that biases them towards one colour interpretation, they mostly retain that interpretation when subsequently viewing the original, more ambiguous image, suggesting a role for one-shot 
learning (Daoudi, Doerig, Parkosadze, Kunchulia, \& Herzog, 2017). However, perceived dress colour is not strongly correlated with the location of first fixation (Daoudi et al., 2017) or chronotype (Aston \& Hurlbert, 2017), so there is still much to learn about the sources of these remarkable individual differences.

\section{Future directions}

The work reviewed here shows that human vision relies heavily on priors and cues to lighting conditions. Nevertheless, there is no standard model of how the visual system represents lighting, and our understanding of how lighting conditions are estimated and used to infer important visual features is tentative and incomplete. Some current models suggest that human vision represents lighting direction and diffuseness (Boyaci et al., 2003; Bloj et al., 2004), and possibly illuminance as well (Logvinenko \& Maloney, 2006), while others claim that we do not represent lighting at all for some purposes (Fleming, Holtmann-Rice, \& Bülthoff, 2011; Gilchrist, 2018). Thus there is a strong need for more general and robust theories of perceptual models of lighting. In recent years it has become easier to carry out experiments to test such theories, as advances in physically based rendering, including models of materials with complex reflectance functions and subsurface scattering, have made it possible to create and manipulate lighting cues in highly realistic stimuli (e.g., Pharr, Jakob, \& Humphreys, 2016). It has also become easier to measure and characterize natural lighting, as devices for measuring illumination maps have advanced from custom-built tools (Debevec, 1998; Mury et al., 2009a; Morgenstern et al., 2014) to off-the-shelf, high-resolution, high dynamic range omnidirectional cameras (e.g., Adams et al., 2016).

A better understanding of perceptual models of lighting would have many applications. Virtual reality (VR) systems are becoming more widely used in many practical settings, but perceptual judgements can be quite different in real and virtual environments (e.g., Morgenstern et al., 2014; but see Radonjić et al., 2016). Fully realistic, physically based rendering is too 
demanding for typical VR systems to perform in real time, so virtual environments must provide viewers with an adequate and more easily computable subset of task-relevant information. Improved models of how human vision represents and estimates lighting conditions would be useful for understanding how computational resources should be allocated to maximize human performance in rendered environments.

A more general theory of perceptual models of lighting would also improve our understanding of several related topics, including lightness and colour constancy, shape from shading, and material perception, in addition to perception of the light field itself (Schirillo, 2013). Lighting perception has received much less attention than other core topics in vision (Gilchrist, 2006, pp. 213-223), which is surprising as it is intrinsically bound up with many of them. Technical developments in experimental methods, new applications, and its central theoretical role make this topic a promising area for advancing our understanding of human vision.

\section{Funding}

This work was supported by a Natural Sciences and Engineering Research Council grant to R.F.M. and an Engineering and Physical Sciences Research Council grant to W.J.A. 


\section{REFERENCES}

Adams, W. J. (2007). A common light-prior for visual search, shape, and reflectance judgments. Journal of Vision, 7(11), 1-7.

Adams, W. J., \& Elder, J.E. (2014). Effects of specular highlights on perceived surface convexity. PLOS Computational Biology, 10(5), 1-13.

Adams, W. J., Elder, J. E., Graf, E. W., Leyland, J., Lugtigheid, A. J., \& Muryy, A. (2016).

The Southampton-York Natural Scenes (SYNS) dataset: statistics of surface attitude. Scientific Reports, 6:35805. * Overview of a publicly available dataset containing high-dynamic range illumination map measurements along with co-registered depth range images

Adams, W. J., Kerrigan, I. S., \& Graf, E. W. (2010). Efficient visual recalibration from either visual or haptic feedback: The importance of being wrong. Journal of Neuroscience, $30(44), 14745-14749$.

Adams, W., Kucukoglu, G., Landy, M., \& Mantiuk, R. (2018). Naturally glossy: gloss perception, illumination statistics and tone mapping. Journal of Vision, 18(3):4, 1-16.

Adams, W. J., Graf, E. W., \& Ernst, M. O. (2004). Experience can change the lightfrom-above' prior. Nature Neuroscience, 7(10), 1057-1058.

Aston, S., \& Hurlbert, A. (2017). What \#thedress reveals about the role of illumination priors in color perception and color constancy. Journal of Vision, 17(9):4, 1-18.

Basri, R., \& Jacobs, D. (2001). Lambertian reflectance and linear subspaces. IEEE Transactions on Pattern Analysis and Machine Intelligence, 25(2), 218-233.

Belhumeur, P. N., Kriegman, D. J., \& Yuille, A. L. (1999). The bas-relief ambiguity. International Journal of Computer Vision, 35(1), 33-44.

Bloj, M., Ripamonti, C., Mitha, K., Hauck, R., Greenwald, S., \& Brainard, D. H. (2004). An equivalent illuminant model for the effect of surface slant on perceived lightness. Journal of Vision, 4(9): 6, 735-746. 
Boyaci, H., Doerschner, K., and Maloney, L. T. (2006a). Cues to an equivalent lighting model, Journal of Vision, 6, 106-118.

Boyaci, H., Maloney, L. T., \& Hersh, S. (2003). The effect of perceived surface orientation on perceived surface albedo in binocularly-viewed scenes. Journal of Vision, 3(8):2, $541-553$.

Brainard, D. H., \& Hurlbert, A. C. (2015). Colour vision: understanding \#thedress. Current Biology, 25, R551-R554. * A brief but critical and wide-ranging overview of possible causes of individual differences in perception of \#thedress

Brox, J. (2011). Brilliant: the evolution of artificial light. Mariner Books.

Brunswik, E., \& Kamiya, J. (1953). Ecological cue-validity of 'proximity' and of other Gestalt factors. American Journal of Psychology, 66, 20-32.

Cronin, T. W., Johnsen, S., Marshall, N. J., \& Warrant, E. J. (2014). Visual ecology. Princeton University Press.

Cuttle, C. (2015). Lighting design: a perception-based approach. Routledge.

Daoudi, L. D., Doerig, A., Parkosadze, K., Kunchulia, M., Herzog, M. H. (2017). The role of one-shot learning in \#thedress. Journal of Vision 17(3):15, 1-7. * Experimentally induced biases in perception of \#thedress are stable and robust; suggests that individual differences may depend on idiosyncratic initial percepts

Debevec, P. (1998). Rendering synthetic objects into real scenes: bridging traditional and image-based graphics with global illumination and high dynamic range photography. In Proceedings of SIGGRAPH 98, Annual Conference Series, pp. 189-198.

Dixon, E. L., \& Shapiro, A. G. (2017). Spatial filtering, color constancy, and the colorchanging dress. Journal of Vision, 17(3):7, 1-20.

Dror, R. O., Willsky, A. S., \& Adelson, E. H. (2004). Statistical characterization of realworld illumination. Journal of Vision, 4(9):11, 821-837. 
Duffy, J. F., \& Czeisler, C. A. (2009). Effect of light on human circadian physiology. Sleep Medicine Clinics, 4(2), 165-177.

Ehinger, K., Adams, W., Graf, E., \& Elder, J. (2017). Local depth edge detection in humans and deep neural networks. IEEE International Conference on Computer Vision Workshops. October 22-29, 2017, Venice, Italy. Los Alamitos, CA: IEEE.

Fleming, R. W., Dror, R. O., \& Adelson, E. H. (2003). Real-world illumination and the perception of surface reflectance properties. Journal of Vision, 3(5), 347-368.

Fleming, R. W., Holtmann-Rice, D., \& Bülthoff, H. H. (2011). Estimation of 3D shape from image orientations. Proceedings of the National Academy of Sciences, 108(51), 2043820443.

Gegenfurtner, K. R., Bloj, M., \& Toscani, M. (2015). The many colours of 'the dress'. Current Biology, 25, R543-R544.

Geisler, W. S. (2008). Visual perception and the statistical properties of natural scenes. Annual Review of Psychology, 59, 167-192.

Gershun, A. (1939). "The light field", translated from Russian by P. Moon and G. Timkoshenko. Journal of Mathematical Physics, 18, 51-151.

Gibson, J. J. (1979). The ecological approach to visual perception. Boston: Houghton Mifflin.

Gilchrist, A., Kossyfidis, C., Bonato, F., Agostini, T., Cataliotti, J., Li, X., ... Economou, E. (1999). An anchoring theory of lightness perception. Psychological Review, 106(4), 795-834. Gilchrist A. L. (1977). Perceived lightness depends on perceived spatial arrangement. Science, 195 (4274), 185-187.

Gilchrist, A. L. (2006). Seeing black and white. Oxford University Press.

Gilchrist, A. L. (2018). To compute lightness, illumination is not estimated, it is held constant. Journal of Experimental Psychology: Human Perception and Performance, 44(8), 1258-1267. 
Granrud, C. E., Yonas, A., \& Opland, E. A. (1985). Infants' sensitivity to the depth cue of shading. Perception \& Psychophysics, 37(5), 415-419.

Hunter, R. S. (1937). Methods of determining gloss. Journal of Research of the National Bureau of Standards, 18, 19-39.

Ikeda, M., Shinoda, H. and Mizokami, Y. (1998). Three dimensionality of the recognized visual space of illumination proved by hidden illumination. Optical Review, 5(3), 200-205.

Kartashova, T., Sekulovski, D., de Ridder, H., te Pas, S.F., \& Pont, S.C. (2016) The global structure of the visual light field and its relation to the physical light field. Journal of Vision 16 (10), 9-9

Kartashova, T., de Ridder, H., te Pas, S. F., \& Pont, S. C. (2018). Visual light Zones. iPerception 9(3).

Kerrigan, I. S., \& Adams, W. J. (2013). Learning different light prior distributions for different contexts. Cognition, 127, 99-104.

Koenderink, J. J., Pont, S. C., van Doorn, A. J., Kappers, A. M. L., \& Todd, J. T. (2007). The visual light field. Perception, 36(11), 1595-1610.

Khan, S., Bennamoun, M., Sohel, F., \& Togneri, R. (2016). Automatic shadow detection and removal from a single image. IEEE Transactions on Pattern Analysis and Machine Intelligence, 38(3), 431-446.

Lafer-Sousa, R., Hermann, K. L., \& Conway, B. R. (2015). Striking individual differences in color perception uncovered by 'the dress' photograph. Current Biology, 25, R545-R546.

Logvinenko, A. D., \& Maloney, L. T. (2006). The proximity structure of achromatic surface colors and the impossibility of asymmetric lightness matching. Perception \& Psychophysics, 68(1), 76-83.

Magnusson, A., \& Boivin, D. (2003). Seasonal affective disorder: an overview. Chronobiology International, 20(2), 189-207. 
Mahroo, O. A., Williams, K. M., Hossain, I. T., Yonova-Doing, E., Kozareva, D., Yusuf, A., ..., Soorma, T. (2017). Do twins share the same dress code? Quantifying relative genetic and environmental contributions to subjective perceptions of "the dress" in a classical twin study. Journal of Vision, 17(1):29, 1-7.

Marlow, P. J., \& Anderson, B. L. (2013). Generative constraints on image cues for perceived gloss. Journal of Vision, 13(14):2, 1-23.

Marlow, P. J., Kim, J., \& Anderson, B. L. (2012). The perception and misperception of specular surface reflectance. Current Biology, 22, 1909-1913.

Metzger, W. (2006). Laws of seeing. Cambridge, MA: MIT Press. (Original work published 1936.)

Morgenstern, Y., Murray, R. F., \& Harris, L. R. (2011). The human visual system's assumption that light comes from above is weak. Proceedings of the National Academy of Sciences, 108(30), 12551-12553.

Morgenstern, Y., Geisler, W. S., \& Murray, R. F. (2014). Human vision is attuned to the diffuseness of natural light. Journal of Vision, 14(9):15, 1-17.

Motoyoshi, I., \& Matoba, H. (2012). Variability in constancy of the perceived surface reflectance across different illumination statistics. Vision Research, 53, 30-39.

Mury, A. A., Pont, S. C., \& Koenderink, J. J. (2007). Light field constancy within natural scenes. Applied Optics, 46, 7308-7316.

Mury, A. A., Pont, S. C., \& Koenderink, J. J. (2009a). Representing the light field in finite three-dimensional spaces from sparse discrete samples. Applied Optics, 48, 450-457.

Mury, A. A., Pont, S. C., \& Koenderink, J. J. (2009b). Structure of light fields in natural scenes. Applied Optics, 48, 5386-5395.

Olkkonen, M., \& Brainard, D. H. (2010). Perceived glossiness and lightness under realworld illumination. Journal of Vision, 10(9):5, 1-19. 
Ostrovsky, Y., Cavanagh, P., \& Sinha, P. (2005). Perceiving illumination inconsistencies in scenes. Perception, 34, 1301-1314.

Pharr, M., Jakob, W, \& Humphreys, G. (2016). Physically based rendering: from theory to implementation (3rd ed.). Morgan Kaufmann.

Pont, S. C., \& te Pas, S. F. (2006). Material- illumination ambiguities and the perception of solid objects. Perception, 35, 1331-1350.

Pont, S. C., \& Koenderink, J. (2007). Matching illumination of solid objects. Perception \& Psychophysics, 69(3), 459-468.

Rabin, J., Houser, B., Talbert, C., \& Patel, R. (2016). Blue-black or white-gold? Early stage processing and the color of 'the dress'. PLOS One, 11(8): e0161090.

Radonjić, A., Pearce, B., Aston, S., Krieger, A., Dubin, H., Cottaris, N. P., Brainard, D. H., \& Hurlbert, A. C. (2016). Illumination discrimination in real and simulated scenes. Journal of Vision, 16(11):2, 1-18.

Ramachandran, V. S. (1988a). Perception of shape from shading. Nature, 331, 163166.

Ramachandran, V. S. (1988b). Perceiving shape from shading. Scientific American, August 1988.

Ramamoorthi, R., \& Hanrahan, P. (2001). On the relationship between radiance and irradiance: determining the illumination from images of a convex Lambertian object. Journal of the Optical Society of America A, 18(10), 2448-2459.

Rutherford, M. D., \& Brainard, D. H. (2002). Lightness constancy: a direct test of the illumination-estimation hypothesis. Psychological Science, 13(2), 142-149.

Schirillo, J. A. (2013). We infer light in space. Psychonomic Bulletin and Review, 20, 905-915.

Snyder, J. L., Doerschner, K., and Maloney, L. T. (2005). Illumination estimation in three-dimensional scenes with and without specular cues. Journal of Vision, 5, 863-877. 
Thomas, R., Nardini, M., \& Mareschal, D. (2010). Interactions between "light-fromabove" and convexity priors in visual development. Journal of Vision, 10(8):6, 1-7.

Toscani, M., Gegenfurtner, K. R., \& Doerschner, K. (2017). Differences in illumination estimation in \#thedress. Journal of Vision, 17(1):40, 1-14.

Uchikawa, K., Morimoto, T., \& Matsumoto, T. (2017). Understanding individual differences in color appearance of \#TheDress based on the optimal color hypothesis. Journal of Vision, 17(8):10, 1-14.

van Doorn, A. J., Koenderink, J. J., \& Wagemans, J. (2011). Light fields and shape from shading. Journal of Vision, 11, 1-21.

van Doorn, A. J., Koenderink, J. J., Todd, J.T. \& Wagemans, J. (2012). Awareness of the light field: the case of deformation. i-Perception, 3, 467-480.

Vemuri, K., Srivastava, A., Agrawal, S., \& Anand, M. (2018). Age, pupil size differences, and color choices for the "dress" and the "jacket". Journal of the Optical Society of America A, 35(4), B347-B355.

Wilder, J. D., Adams, W. J., \& Murray, R. F. (2019). Shape from shading under inconsistent illumination. Journal of Vision, 19(6):2, 1-15.

Winkler, A. D., Spillmann, L., Werner, J. S., \& Webster, M. A. (2015). Asymmetries in blue-yellow color perception and in the color of 'the dress'. Current Biology, 25, R547-R548.

Witzel, C., Racey, C., \& O'Regan, J. K. (2017). The most reasonable explanation of "the dress": implicit assumptions about illumination. Journal of Vision, 17(2):1, 1-19.

Xia, L., Pont, S. C., \& Heynderickx, I. (2014). The visual light field in real scenes. $i$ Perception, 5, 613-629.

Xia, L., Pont, S. C., \& Heynderickx, I. (2017a). Light diffuse metric, Part 1: Theory. Lighting Research and Technology, 49: 411-427. * Examines several measures of lighting diffuseness; exemplary use of illumination map methods to quantify a perceptually important property of lighting 
Xia, L., Pont, S. C., \& Heynderickx, I. (2017b). Light diffuse metric, Part 2: Describing, measuring and visualising the light flow and diffuseness in three-dimensional spaces. Lighting Research and Technology, 49: 428-445.

Xia, L., Pont, S. C., \& Heynderickx, I. (2017c). Separate and simultaneous adjustment of light qualities in a real scene. i-Perception, 8(1). * Evaluates human performance in judging basic properties of lighting; shows that making lighting settings on a spherical probe is an accurate and practical way of evaluating lighting percepts 


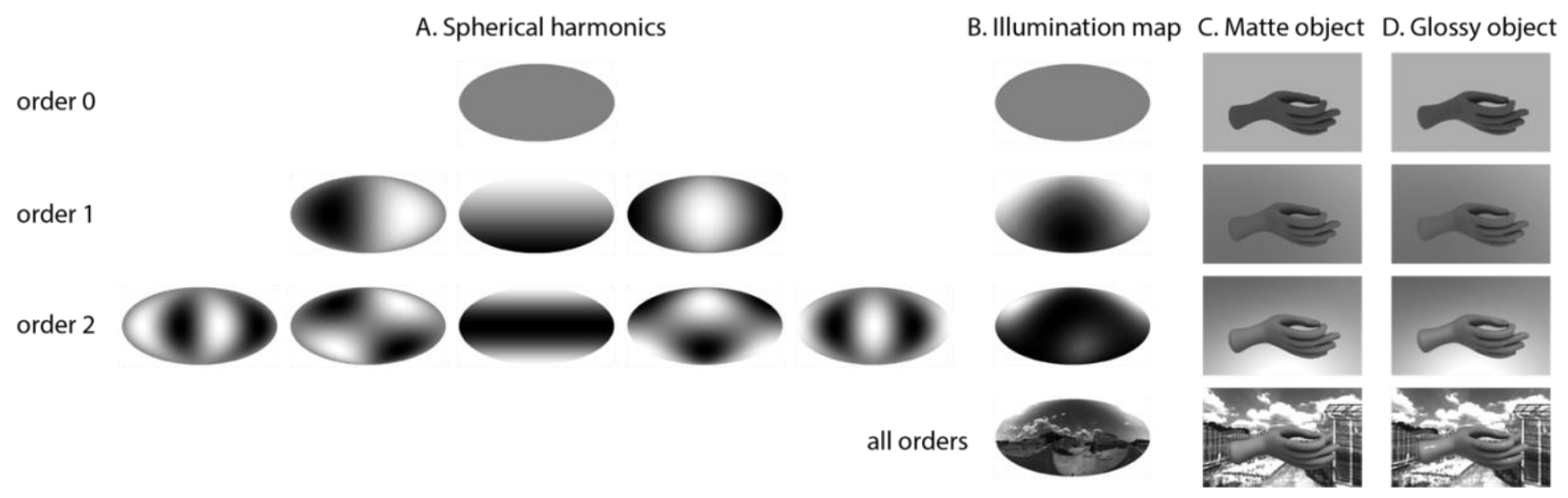

Figure 1. (A) Real-valued spherical harmonics up to order 2, shown in Mollweide projection.

(B) Approximations to an illumination map that include all spherical harmonics up to orders 0, 1, and 2, as well as the full illumination map. (C) A matte object and (D) a glossy object rendered with the illumination maps in column (B). 

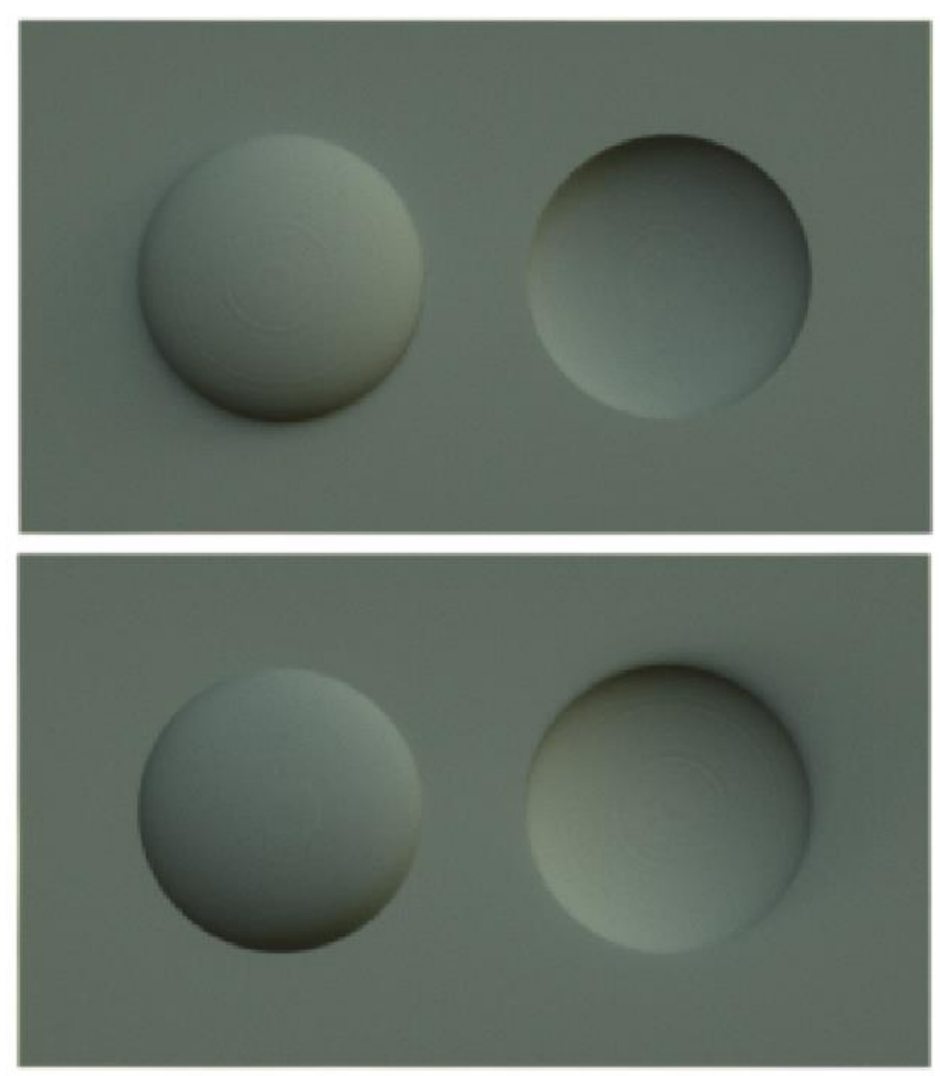

Figure 2. Simple objects rendered under natural illumination. To most observers, objects on the left appear convex, and those on the right as concave. However, in the lower row the lightfrom-above prior competes with information from cast shadows (caused by illumination from below) making perceived shape more ambiguous: the lower right object might appear convex. 


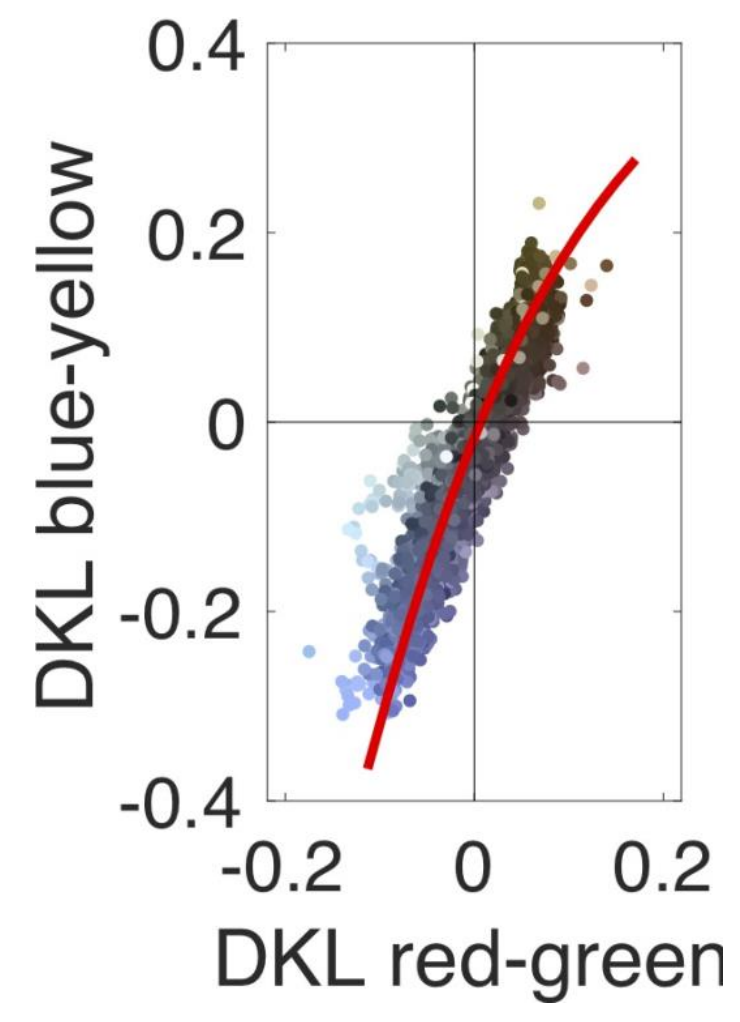

Figure 3. Pixelwise chromaticity of \#thedress in DKL colour space. The red line shows the daylight locus. (From Gegenfurtner et al., 2015) 